\title{
EchoGéo
}

$32 \mid 2015$

Inde : le grand écart spatial

\section{Différenciations territoriales et insertion dans la mondialisation : le cas de l'énergie en Inde}

Nicolas Mazzucchi

\section{(2) OpenEdition}

\section{Journals}

Édition électronique

URL : https://journals.openedition.org/echogeo/14259

DOI : 10.4000/echogeo.14259

ISSN : 1963-1197

Éditeur

Pôle de recherche pour l'organisation et la diffusion de l'information géographique (CNRS UMR 8586)

Référence électronique

Nicolas Mazzucchi, « Différenciations territoriales et insertion dans la mondialisation : le cas de l'énergie en Inde », EchoGéo [En ligne], 32 | 2015, mis en ligne le 15 juillet 2015, consulté le 11 août 2021. URL : http://journals.openedition.org/echogeo/14259; DOI : https://doi.org/10.4000/echogeo. 14259

Ce document a été généré automatiquement le 11 août 2021.

EchoGéo est mis à disposition selon les termes de la licence Creative Commons Attribution - Pas d'Utilisation Commerciale - Pas de Modification 4.0 International (CC BY-NC-ND) 


\title{
Différenciations territoriales et insertion dans la mondialisation : le cas de l'énergie en Inde
}

\author{
Nicolas Mazzucchi
}

1 L'Inde est devenue en l'espace de quelques décennies l'une des économies les plus dynamiques de la planète. Passant du $16^{\mathrm{e}}$ rang mondial en termes de PIB en $1995 \mathrm{au}$ $8^{\mathrm{e}}$ rang aujourd'hui, elle fait partie des puissances dites émergentes au même titre que la Chine ou le Brésil. Toutefois cette croissance économique, de l'ordre de $5 \%$ en 2013 (8,3\% de moyenne annuelle sur la période 2003-2013), engendre elle-même de nombreux défis aussi bien politiques qu'économiques pour la puissance d'Asie du sud. Le premier et, sans doute, le plus complexe d'entre eux tient à la nécessité pour l'Inde d'alimenter en énergie tant ses entreprises que sa population, elle-même en croissance continue. La question énergétique représente actuellement pour New Delhi la principale problématique politico-économique et un enjeu majeur de ce début de $\mathrm{XXI}^{\mathrm{e}}$ siècle. Les déterminants de l'équation énergétique semblent particulièrement délicats à gérer. En effet l'Inde fait face à des difficultés et doit arbitrer entre des priorités nationales et locales très contrastées. Ceci se traduit tant pour l'accès à l'énergie ${ }^{1}$ pour la sécurité énergétique ${ }^{2}$ et, depuis quelques années, la lutte contre le changement climatique.

2 Le développement national laisse apparaitre des fractures régionales avec de profondes différences entre les États fédérés, que ce soit au niveau social ou économique. La question énergétique est un révélateur majeur des disparités. Ces dernières induisent des priorités différentes avec un délicat équilibrage à accomplir au niveau national. Ainsi les choix locaux ont obligatoirement des répercussions sur la politique énergétique du pays. En outre, celle-ci est conditionnée à des questions technologiques. L'histoire complexe du développement énergétique du pays, surtout vis-à-vis du nucléaire civil, a entrainé l'apparition d'entreprises autochtones dans ce domaine, qui dominent le secteur. Or les besoins nationaux et les enjeux mondiaux liés à la lutte contre le changement climatique obligent de plus en plus l'Inde à avoir recours à des 
solutions technologiques étrangères. Cette nouvelle donne de l'énergie modifie grandement les pratiques nationales. Elle pourrait à terme bouleverser tout le secteur et, avec lui, une partie non négligeable de la politique étrangère indienne, sans même parler du risque d'une rupture de l'équilibre national. L'analyse des besoins nationaux en énergie, au travers principalement des sources officielles et entrepreneuriales, en lien avec les évolutions de la population indienne et de l'économie, laisse entrevoir l'équilibre difficile entre le niveau local et le jeu du marché global, révélant l'augmentation des disparités entre les différents États indiens.

\section{Le secteur énergétique national}

3 Les besoins nationaux en matière énergétique ne cessent d'augmenter à cause d'une double évolution structurelle du pays. D'un côté l'augmentation continue de la population indienne induit une hausse de la demande énergétique nationale. D'un autre côté l'évolution qualitative de la demande, poussée par une transformation de la société vers des modèles de consommation plus «développés » - à l'instar de ce qui se passe en Chine depuis le début des années 2000 - augmente la demande énergétique par habitant. Outre une hausse de la demande domestique, l'industrialisation en cours et le développement du secteur des services - par exemple dans l'informatique - ont également un impact important sur la projection de la demande. Cette double hausse, qualitative et quantitative, se retrouve dans l'ensemble des pays émergents et conditionne profondément l'évolution de l'ensemble du secteur énergétique mondial. Celle-ci induit une compétition entre États et entreprises pour l'accès aux ressources aussi bien dans le domaine des hydrocarbures que dans celui des métaux stratégiques, particulièrement importants dans le domaine des énergies renouvelables et du nucléaire.

Ainsi l'Inde se trouve obligée de s'insérer dans une concurrence internationale de plus en plus féroce pour les matières premières. Celle-ci est d'autant plus importante que le pays est mal doté en ressources fossiles par rapport à ses besoins, ce qui en fait l'un des principaux importateurs mondiaux d'hydrocarbures. L'absence d'importantes matières premières pétrolières ou gazières nationales, malgré de récentes découvertes de ressources gazières, a obligé le pays à se tourner vers les mines de charbon pour son développement économique. Cette utilisation massive est notamment due à la grande densité de population $\left(368 \mathrm{hab} / \mathrm{km}^{2}\right)$ sur un territoire finalement assez restreint, imposant le recours à de grandes unités de production peu coûteuses. Le charbon représente toujours la base de la consommation du pays avec près de $45 \% \mathrm{du}$ mix énergétique national (illustration 1). Ce dernier est complété par le pétrole, base du secteur des transports, mais aussi par une part importante de biomasse/déchets manifestant l'importance locale de l'utilisation des sous-produits domestiques (résidus ménagers ou animaux comme la bouse séchée) et industriels ainsi que de bois. Le mix énergétique indien est ainsi marqué par une utilisation importante de ressources peu transformées et fortement émettrices de gaz à effet de serre, alors que les énergies plus avancées technologiquement comme le nucléaire ou les énergies renouvelables (ENR) dites de «nouvelle génération " (éolien, solaire, géothermique, petit hydraulique, etc.) n'occupent qu'une place très secondaire avec moins de $3 \%$ du mix énergétique national. La croissante effrénée dans les domaines économique et démographique a imposé une adaptation rapide du secteur énergétique avec un risque structurel pour ce 
dernier, écartelé entre les questions d'accès à l'énergie et de sécurité des approvisionnements.

Illustration 1 - Mix énergétique indien (2012)

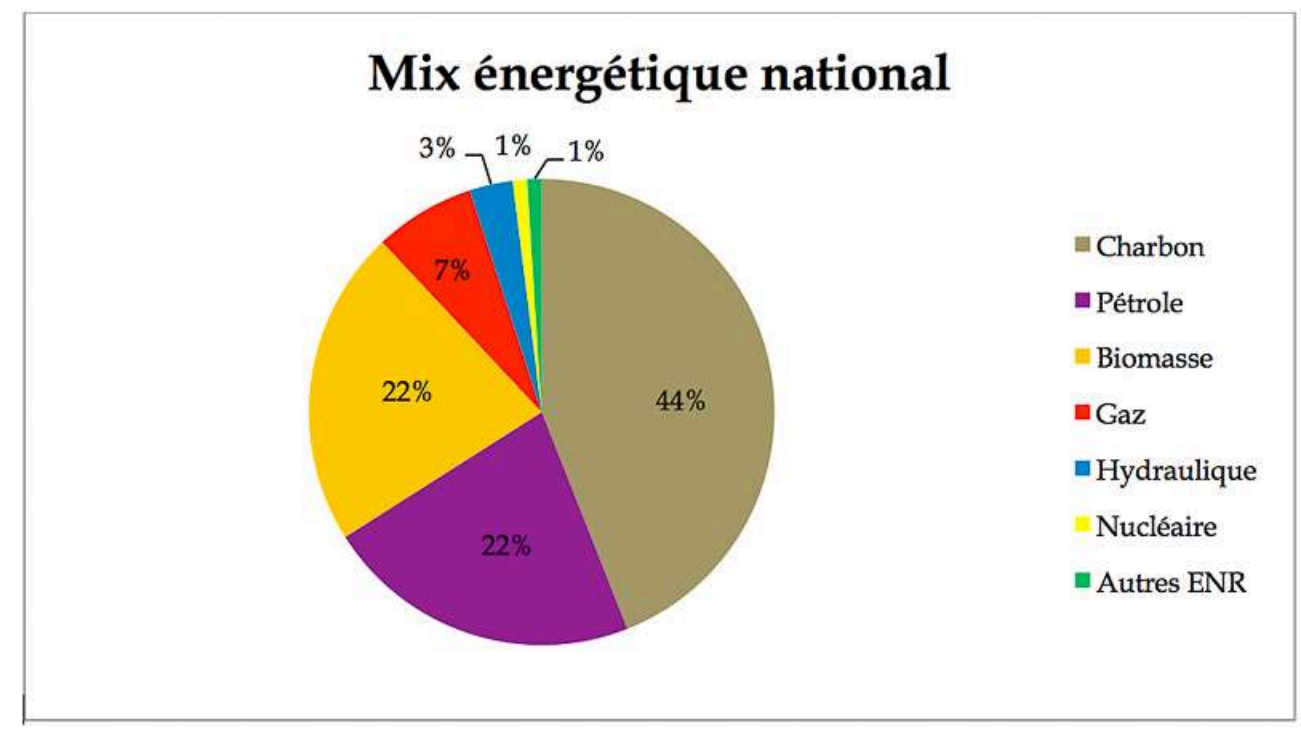

Source : Energy Information Administration.

5 La hausse des émissions de $\mathrm{CO}_{2}$ du pays ont positionné l'Inde comme l'un des pays les plus pollueurs de la planète. Le Global Carbon Atlas positionne l'Inde comme le $3^{e}$ émetteur de $\mathrm{CO}_{2}$ au niveau mondial avec 2407 millions de tonnes en 2013, même si son taux d'émissions par habitant (1,9 tonne) reste limité par rapport aux pays du Nord (16 tonnes pour les États-Unis) ou à la Chine (7,2 tonnes) $)^{3}$. L'Inde est ainsi l'un des principaux émetteurs de gaz à effet de serre de la planète, ce qui explique largement son positionnement particulièrement ferme lors des dernières conférences climatiques de la Convention Cadre des Nations-Unies sur le Changement Climatique. À titre d'exemple, lors de la conférence COP 15 de Copenhague de 2009, l'Inde a adopté une position plus dure encore que celle de la Chine, ne proposant qu'une réduction de $15 \%$ de l'intensité carbone du pays. Eu égard aux trois facteurs que sont la croissance économique nationale, l'utilisation massive de charbon pour la production électrique et le retard technologique dans les énergies renouvelables, l'Inde se trouve dans une position délicate (illustration 2). Accepter de faire des efforts importants pour la protection du climat, passant éventuellement par l'instauration d'une taxe carbone, obérerait nécessairement la croissance économique, mettant en péril l'équilibre socioéconomique national. La lutte contre le changement climatique semble ainsi être mise en retrait par rapport aux éléments liés à la production et à la sécurisation des approvisionnements en énergie. 
Illustration 2 : Évolution des émissions de $\mathrm{CO} 2$ de l'Inde en milliers de tonnes

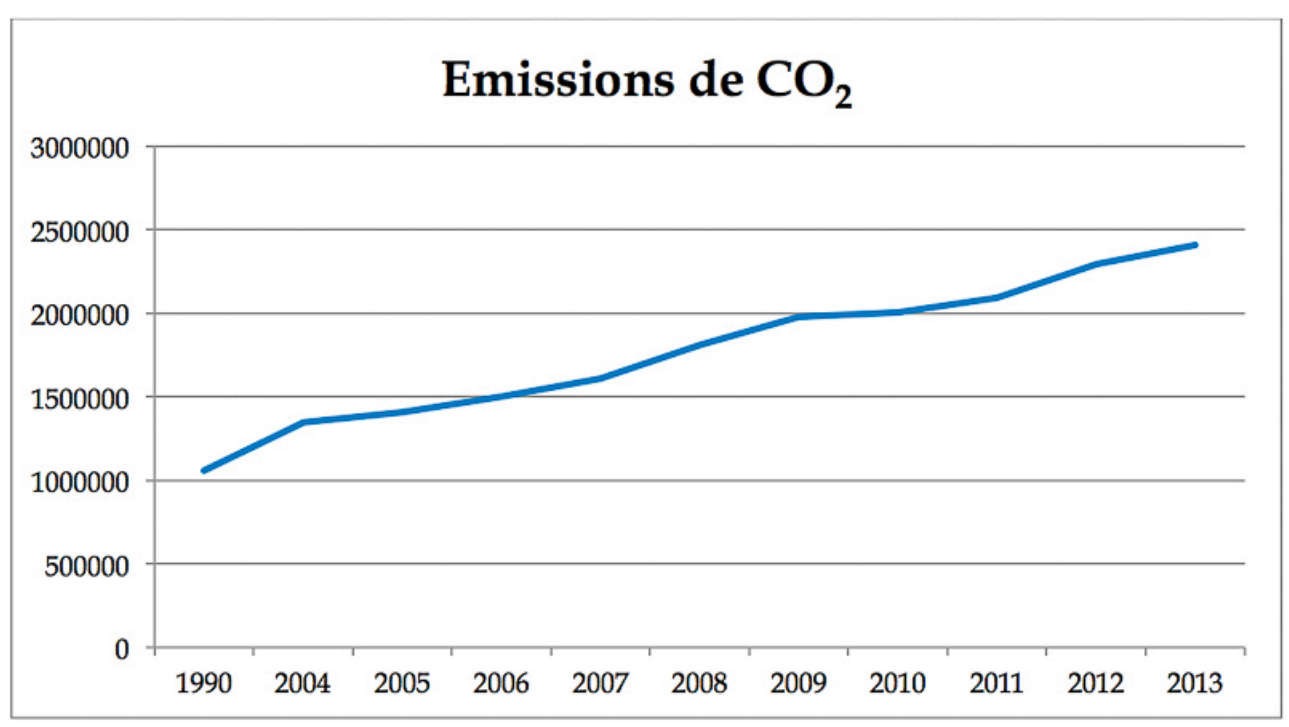

Source : Banque Mondiale; Global Carbon Atlas.

La dépendance de l'Inde aux hydrocarbures, très majoritairement en pétrole dont l'Inde est le $4^{\mathrm{e}}$ importateur mondial ${ }^{4}$, que ce soit pour la production électrique et surtout pour le transport crée un ancrage géopolitique particulier. Le pays s'approvisionne en majorité au Moyen-Orient, avec l'Iran, mais aussi l'Irak ou l'Arabie saoudite. Au niveau gazier également les appétits de l'Inde ne cessent de se développer. Alors que le pays n'importait pas de gaz naturel liquéfié (GNL) avant 2004, il en est devenu en l'espace d'une décennie le $4^{\mathrm{e}}$ importateur mondial. L'Inde se trouve ainsi, nolens volens, impliquée dans les problèmes géopolitiques du Moyen-Orient puisque les soubresauts de cette région, comme le chaos irakien ou la diminution constante de la production iranienne suite aux embargos occidentaux, ont des répercussions sur la sécurité des approvisionnements nationaux. Toutefois l'Inde ne semble pas vouloir s'impliquer dans la dynamique conflictogène d'une région particulièrement instable et diversifie ses approvisionnements entre des acteurs diamétralement opposés sur l'échiquier géopolitique (Arabie saoudite, Iran, Irak, etc.) pour limiter son attachement à l'un plutôt qu'à l'autre. De plus les limites de la capacité militaire et surtout diplomatique du pays, eu égard aux relations passablement tendues avec ses voisins immédiats, imposent des contraintes supplémentaires en termes de sécurisation des approvisionnements. Dans ce cadre les projets internationaux de grandes infrastructures comme le gazoduc TAPI (Turkménistan-Afghanistan-Pakistan-Inde), porté au début des années 2000 par l'américain Unocal et depuis quelques années par le chinois CNPC, s'ils permettent d'envisager une plus grande diversité des fournisseurs, mettent également le pays sous la dépendance du Pakistan - comme pays de transit voire de la Chine - comme possesseur de l'infrastructure.

\section{Marché mondialisé contre besoins locaux}

7 L'augmentation de la demande énergétique nationale entraîne une insertion de plus en plus importante de l'État et des entreprises indiennes sur le marché énergétique mondial. Les besoins du pays en charbon sont tels que les exploitations indiennes 
n'arrivent plus à satisfaire la demande et qu'on a recours aux importations depuis l'Indonésie ou l'Australie. L'Inde qui est le $3^{\mathrm{e}}$ plus gros consommateur de charbon au monde derrière la Chine et les États-Unis, se trouve - là-aussi - obligée de penser sa sécurité énergétique en termes de sécurisation des flux d'approvisionnements malgré les immenses réserves détenues.

Cette stratégie axée sur les ressources étrangères est de plus en plus prégnante, au point que les entreprises publiques du secteur pétro-gazier comme ONGC deviennent progressivement actives sur les marchés étrangers y compris dans l'explorationproduction. Ainsi ONGC via sa filiale ONGC Videsh est-elle implantée dans de nombreux projets en Russie (Sakhaline-1), en Birmanie, au Vietnam, au Venezuela (San Cristobal), au Sud-Soudan, en Azerbaïdjan ou au Brésil. OIL de son côté est présente en Libye, au Gabon, au Nigeria, en Russie, au Venezuela ou en Birmanie. L'internationalisation de ces entreprises s'est effectuée, de la même manière que pour Petrobras au Brésil (Mazzucchi, 2012), par un besoin croissant de sécuriser les approvisionnements nationaux depuis la phase amont de la chaine de valeur pétrolière. La hausse continue de la demande en pétrole et surtout en gaz naturel, a progressivement obligé les compagnies nationales publiques à signer des accords avec des États pétro-gaziers ou leurs entreprises dans le but de participer directement à la production.

Cette stratégie va de pair avec un défi technologique plus poussé. Les terminaux de regazéification de Dahej et Kochi sont certes possédés et opérés par la compagnie nationale Petronet LNG, mais cette dernière est une joint-venture entre les compagnies nationales pétrolières (ONGC, OIL) et gazières (GAIL) et des partenaires étrangers comme GDF-Suez et la Banque Asiatique de Développement. En outre, le terminal de Hazira, le $2^{\mathrm{e}}$ plus important du pays, est possédé conjointement par Shell et Total. La nécessité d'implanter ces nouvelles infrastructures logistiques oblige à ouvrir de plus en plus le secteur aux majors occidentales pour bénéficier des technologies de ces dernières.

Le besoin énergétique tous azimuts de l'Inde amène New Delhi à faire flèche de tout bois et à se tourner tant vers la recherche forcenée de sources d'approvisionnement fiables en hydrocarbures que vers un développement technologique, nucléaire et dans une moindre mesure vers les énergies renouvelables. Ainsi, les choix de l'Inde en matière nucléaire s'expliquent avant tout par une volonté farouche de se rapprocher un peu plus de l'indépendance énergétique nationale. La volonté nucléaire naît également d'une recherche de l'image de "puissance" par les dirigeants du pays. L'histoire nucléaire de l'Inde commence avant même l'Indépendance avec les travaux de $\mathrm{H}$. Bhabha, le père de l'atome indien, soutenu financièrement par la famille Tata (Lambert, 2007). Ce dernier envisageait déjà l'énergie atomique non seulement comme une capacité de défense mais également comme un formidable levier de développement social et économique pour son pays (Vinod Kumar, 2014). L'Inde, après avoir construit son propre réacteur de recherche en 1956, bénéficia du programme américain Atoms for peace et, par-là, de la coopération américaine et canadienne pour le développement de centrales. Toutefois la coopération connaît un coup d'arrêt brutal en 1974 lors de la première explosion atomique qui fait entrer le pays dans le club des puissances nucléaires militaires. N'ayant pas signé le Traité de Non-Prolifération (TNP), l'Inde est de facto privée de toute coopération civile. La levée des sanctions américaines en 2008, qui avaient été imposées suite aux essais de 1998, ouvre à nouveau d'importantes perspectives de développement dans ce secteur. Cette situation inédite positionne 
l'Inde comme un des principaux territoires de développement du nucléaire civil pour ce début de $\mathrm{XXI}^{\mathrm{e}}$ siècle. Le pays doit maintenant, au regard de ses besoins et de ses ambitions, avoir recours à l'importation de la technologie des grands acteurs du nucléaire mondiaux (Rosatom, Areva, Toshiba-Westinghouse ou GE-Hitachi).

Le choix des États-Unis de retisser des liens nucléaires avec l'Inde manifeste la volonté de profiter d'un marché qui s'annonce très important pour les entreprises du secteur. Les autorités russes ont, quant à elles, tout à fait intégré ce paramètre et ont proposé depuis plusieurs années leurs savoir-faire en ce domaine. La corporation d'État Rosatom, pilier de la politique nucléaire russe, est ainsi en pointe parmi les entreprises étrangères pour le développement du secteur nucléaire civil indien. Alors que jusqu'au milieu des années 2000 l'Inde s'était, par nécessité plus que par choix, orientée vers des solutions autochtones ${ }^{5}$, l'entrée de Rosatom concrétisée par le contrat de la centrale de Kudankulam ${ }^{6}$, dont la signature a pris plusieurs décennies (1988-2002), marque un changement de paradigme à ce sujet avec le recours à une entreprise étrangère intégrée verticalement. Le partenariat économique russo-indien qui plonge ses racines dans la seconde moitié du $\mathrm{XX}^{\mathrm{e}}$ siècle ${ }^{7}$ reste particulièrement important et New Delhi demeure un interlocuteur privilégié de Moscou sur les questions énergétiques. Moscou apparait également comme un partenaire idéal à cause de sa position de producteur de combustible nucléaire civil. Les réserves russes - auxquelles il faut ajouter le partenariat entre Rosatom et Kazatomprom au Kazakhstan pour l'uranium permettent à Rosatom de fournir l'ensemble des services associés à la chaîne de valeur du nucléaire civil. En outre, l'accord entre Rosatom et l'AIEA concernant le centre international d'enrichissement d'Angarsk inauguré en 2010 permet de disposer d'un combustible agréé et contrôlé par l'organisation onusienne. Cet accord permet à l'Inde de disposer enfin d'approvisionnements de combustible à la hauteur de ses besoins ${ }^{8}$.

Le développement des énergies renouvelables (ENR) en Inde peut quant à lui être une solution à la fragmentation territoriale aussi bien qu'à la question de la préservation de l'environnement. Jusqu'ici le pays avait pâti de la relative polarisation de ses activités économiques les plus énergivores dans la région du nord-ouest, entrainant un développement énergétique à deux vitesses. Or les différents niveaux de puissance des installations ENR de nouvelle génération comme le solaire ou l'éolien, de l'ordre de quelques centaines de MW, les rendent adaptées à une approche plus régionalisée de la politique énergétique. Toutefois ce déploiement en masse des ENR suppose également le recours à des partenaires étrangers occidentaux, japonais et surtout chinois selon les énergies qui seraient choisies. En effet le retard technologique des acteurs nationaux est encore plus important que pour les autres secteurs et cela constituerait donc un cas de dépendance tout à fait nouveau puisque s'inscrivant au niveau des petites infrastructures de production. Dans le domaine du solaire par exemple, la volonté nationale marquée par la Jawaharlal Nehru National Solar Mission (JNNSM) met en avant les limites de la capacité nationale en ce domaine. Si la JNNSM prévoit le déploiement d'ici 2022 de $20 \mathrm{GW}$ de centrales solaires, elle impose aussi qu'au moins la moitié des panneaux soient réalisés en Inde ce qui est impossible eu égard à la faiblesse $\mathrm{du}$ secteur. En conséquence, le programme prend du retard puisque les acteurs nationaux ne sont pas capables de répondre à la demande. En outre, les entreprises américaines du solaire ont posé un recours devant l'OMC en 2013 (affaire DS456) pour demander la levée de cette restriction assimilée à du protectionnisme. Si elles obtenaient gain de cause, cela bénéficierait majoritairement aux acteurs chinois et américains qui deviendraient rapidement incontournables dans le secteur indien des 
ENR. Au niveau de l'énergie éolienne néanmoins, l'Inde semble mieux armée. Avec l'entreprise nationale Suzlon comme porte-étendard ${ }^{9}$, le pays a su développer un vrai secteur éolien et implanter de nombreux parcs dans plusieurs États. Toutefois, à l'exception du Tamil Nadu, l'essentiel de la capacité de production éolienne se trouve majoritairement concentrée dans le quart nord-ouest du pays (Gujarat, Rajasthan, Maharashtra). Cette disparité géographique constatée dans l'éolien se retrouve en fait dans l'ensemble du secteur énergétique indien.

\section{Les disparités des États en matière d'énergie}

$13 \mathrm{Au}$ niveau énergétique comme sur d'autres aspects (population, production de richesses, niveau de maturité économique, etc.), les États fédérés de l'Union indienne offrent une mosaïque de situations contrastées. Le taux national d'accès à l'électricité reste relativement faible, de l'ordre de $75 \%$ en 2011 selon la Banque Mondiale, ce qui place l'Inde au niveau de pays comme l'Indonésie, le Ghana ou le Nicaragua. Le pays accuse ici un retard marqué sur les autres grands émergents, y compris l'Afrique du Sud qui dépasse les $80 \%$. D'autre part ce taux national ne saurait masquer les grandes disparités entre les différents États. Le pays souffre en effet d'une véritable fracture énergétique entre les États les plus avancés et industrialisés et les États les plus pauvres et ruraux. Le grand écart spatial qui existe entre les différents territoires indiens amène à prendre en compte différemment la problématique énergétique. La relative concentration économique nationale de la richesse dans le nord-ouest du pays a sa traduction dans le taux d'accès à l'énergie comme dans l'importance de la capacité de production électrique. Les États les plus riches comme le Maharashtra, l'Uttar Pradesh ou le Gujarat disposent ainsi d'une importante capacité de production d'électricité, allant de pair avec un accès à l'énergie plus développé pour la population statistiquement sinon réellement sur le terrain. La disparité entre le Karnataka et le Gujarat qui ont un niveau de population similaire mais une différence assez marquée dans l'économie nationale reflète bien cette situation. Les rapports de l'Autorité électrique centrale laissent entrevoir un déséquilibre régional en Inde entre un bloc Nord et Ouest relativement bien approvisionné en électricité et des régions Sud et Nord-Est faisant face à un déficit chronique, surtout lors de pics de demande (Central Electric Authority, 2014).

Illustration 3 - Approvisionnements électriques des différentes régions indiennes

\begin{tabular}{|l|l|l|}
\hline Région & $\begin{array}{l}\text { Surplus ou déficit dans la fourniture } \\
\text { électrique en temps normal (\%) }\end{array}$ & $\begin{array}{l}\text { Surplus ou déficit dans la fourniture } \\
\text { électrique lors des pics de demande (\%) }\end{array}$ \\
\hline Nord & $-3,1$ & $-1,4$ \\
\hline Ouest & 0,3 & 14,5 \\
\hline Sud & $-12,7$ & $-22,2$ \\
\hline Est & $-3,4$ & 1 \\
\hline
\end{tabular}




\begin{tabular}{|l|l|l|}
\hline $\begin{array}{l}\text { Nord- } \\
\text { est }\end{array}$ & $-17,4$ & $-12,9$ \\
\hline $\begin{array}{l}\text { Total } \\
\text { Inde }\end{array}$ & $-5,1$ & $-2,0$ \\
\hline
\end{tabular}

Source : Central Electric Authority, 2014.

14 Il existe ainsi une dorsale Mumbai - Delhi qui concentre la plus grande partie de la demande énergétique nationale. Cette dernière correspond au cœur de l'industrie et des services du pays - à l'exception de certaines régions méridionales - reliant la capitale économique à la capitale administrative du pays. C'est largement dans cette dorsale que se joue l'avenir de l'économie indienne et, avec elle, le développement de l'ensemble du pays. Les six États du Gujarat, Maharashtra, Madhya Pradesh, Rajasthan, Uttar Pradesh, Haryana (plus Delhi) représentent à eux seuls près de la moitié $(46,2 \%)$ du PIB et de la capacité de production électrique $(49,1 \%)$ de l'Inde. L'existence d'une telle dorsale du développement se lit également au travers de l'implantation des grandes unités de production électrique du pays. Les centrales nucléaires indiennes, issues du programme national et gérées par l'opérateur national NPCIL, détenu par l'État fédéral, se concentrent principalement dans les États du Gujarat (Kakrapar), du Rajasthan (Rawatbatha), d'Uttar Pradesh (Narora) et du Maharastra (Tarapur).

Illustration 4 - Principales installations énergétiques en Inde

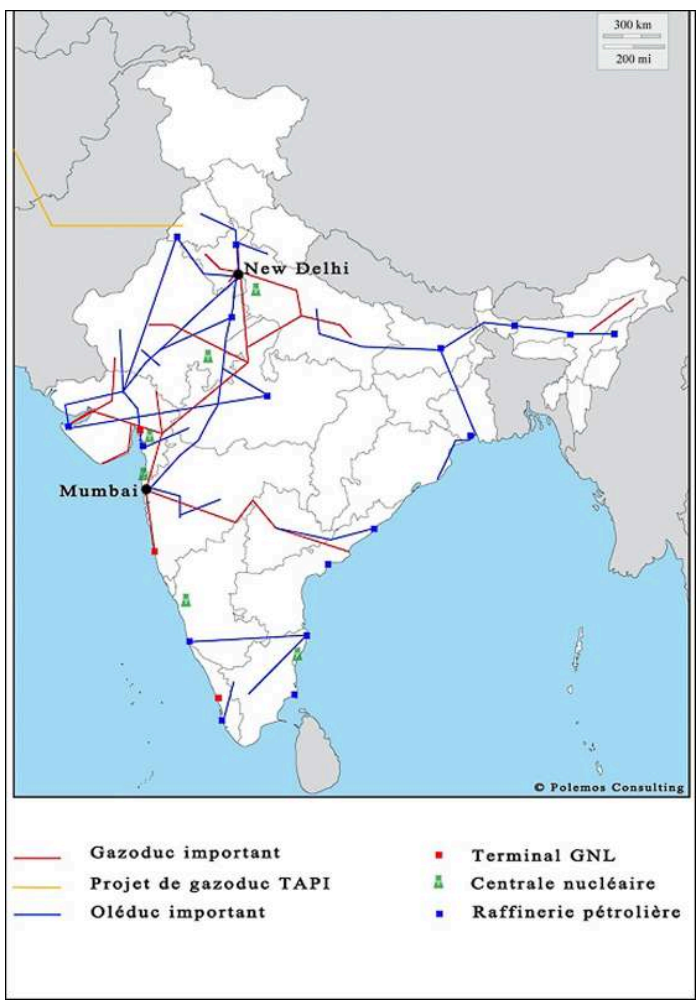


Illustration 5 - Statistiques économiques et énergétiques par État

\begin{tabular}{|l|l|l|l|}
\hline État & $\begin{array}{l}\text { Population en 2011 } \\
\text { (hab.) }\end{array}$ & $\begin{array}{l}\text { Part du PIB national en } \\
\text { 2013 (\%) }\end{array}$ & $\begin{array}{l}\text { Capacité de production } \\
\text { électrique (MW) }\end{array}$ \\
\hline $\begin{array}{l}\text { Uttar } \\
\text { Pradesh }\end{array}$ & 199581477 & 8,2 & 14079 \\
\hline Mahārāshtra & 112372972 & 14,6 & 31934 \\
\hline Bihār & 103804637 & 3,3 & 1868 \\
\hline Karnataka & 61130704 & 5,6 & 13818 \\
\hline Gujarat & 60383628 & 7,1 & 26126 \\
\hline Jharkhand & 32966238 & 1,8 & 2539 \\
\hline Assam & 31169272 & 1,5 & 1140 \\
\hline
\end{tabular}

Sources: recensement national 2011 ; Commission de la planification ; ministère de l'Electricité.

15 Au niveau pétro-gazier, le pays est également marqué par une forte disproportion des infrastructures selon les États. En effet, l'ouest du pays, notamment la région Mumbai Delhi regroupe ici aussi l'essentiel des pipelines, oléoducs et gazoducs, qui relient les principaux centres régionaux aux ports côtiers. Les raffineries pétrolières sont également concentrées suivant cet axe, avec une importante industrie pétrochimique autour de la capitale. Les infrastructures gazières sont encore plus révélatrices de cette orientation puisque la quasi-totalité des gazoducs existants se concentre dans le nordouest du pays avec une répartition des terminaux GNL autour de Mumbai et un réseau d'approvisionnement couvrant principalement la dorsale Mumbai- Delhi. L'inégalité de traitement des territoires se mesure ainsi à leur accès privilégié ou non à l'énergie, le plus souvent fossile. Cet accès s'entend toutefois majoritairement dans un but de satisfaction de la demande industrielle plus que domestique. En ce sens les réseaux de conduites traduisent dans leur géographie d'implantation une organisation politicoéconomique faisant la part belle à certaines zones plus qu'à d'autres. La profonde dichotomie existant au niveau logistique entre les deux côtes de l'Inde s'explique par la localisation des sources d'importation comme des ressources nationales: d'une part l'essentiel du gaz importé provient du Golfe persique. D'autre part, c'est sur cette façade, au large du Gujarat et du Rajasthan que se concentre l'essentiel des réserves pétrolières offshore du pays.

La différence observée entre les territoires manifeste un peu plus de l'inégalité dans le niveau de développement des différentes régions du pays. L'Inde se trouve ainsi confrontée à des problématiques énergétiques qui diffèrent selon les territoires. Si dans les États traversés par la dorsale Mumbai -Delhi (Maharashtra, Gujarat, Madhya Pradesh, Rajasthan, Uttar Pradesh, Haryana) le principal enjeu semble être la sécurité énergétique, dans les États éloignés comme ceux de la partie orientale du pays, l'urgence se manifeste plus au niveau de l'accès à l'énergie au vu de la faiblesse des infrastructures de production électrique notamment. Certes ces deux problématiques 
cohabitent souvent sur même territoire, mais force est de reconnaitre qu'elles peuvent être plus ou moins fondamentales selon les régions. La détermination d'un mix énergétique national apparaît également fort complexe en raison de différences entre territoires. De fait, le développement d'installations technologiquement avancées dans certains États, comme les centrales nucléaires, s'oppose à la présence massive de petites centrales au charbon, au diesel ou à biomasse faisant de l'Inde le pays des extrêmes en matière de production électrique. La coexistence entre les technologies de pointe et les installations anciennes et peu efficientes est un révélateur particulièrement pertinent de la différence entre les États.

$17 \mathrm{Au}$ niveau politique, la dispersion des compétences entre plusieurs niveaux décisionnels renforce également cette différence entre les territoires. Au niveau du gouvernement fédéral la question énergétique est éclatée entre plusieurs entités ministérielles sectorielles (ministères des Énergies nouvelles et renouvelables, du Charbon, du Pétrole et du Gaz naturel, de l'Électricité, de l'Environnement et de la Forêt) et d'autres institutions (Département de l'Énergie atomique, Commission de planification). Cette dispersion des décisions engendre nécessairement une complexité administrative et des lenteurs dans la décision et l'application d'une politique nationale concertée. De plus, la fragmentation des compétences politiques s'entend également dans une approche verticale puisque les États fédérés détiennent constitutionnellement des responsabilités dans la question des réseaux gaziers, de la fixation des prix de l'énergie et de l'usage des terres et de l'eau. En outre, des compétences sont partagées entre les États et le gouvernement central pour la production et de l'acheminement de l'électricité. Cette complexité administrative qui entraîne de manière quasi systématique des conflits de compétence entre les diverses entités, freine l'adoption de nouvelles règlementations et empêche une vision globale des problématiques sectorielles.

\section{Conclusion}

Les croissances démographique et économique de l'Inde représentent des opportunités mais aussi des risques pour la stabilité du pays. Ce dernier se trouve depuis quelques années en pleine transition énergétique. Toutefois celle-ci ne s'effectue pas partout de la même manière et les territoires les plus dynamiques économiquement, regroupés dans la dorsale Mumbai- Delhi, ont une avance technologique bien supérieure à celle des espaces ruraux du Nord ou de l'Est. Cela leur permet de disposer d'un accès continu à l'énergie mais aussi de tenter de limiter de plus en plus les taux de rejet en gaz à effet de serre à production constante ${ }^{10}$. Cette différenciation territoriale semble d'ailleurs s'accélérer avec des modes d'insertion particuliers dans les circuits mondialisés, traduits par l'empreinte logistique des réseaux de pipelines ou de terminaux GNL. Dans l'énergie comme dans de nombreux domaines, la réticulation internationale apparaît comme une marque d'insertion dans la mondialisation. Au-delà même de cette problématique logistique, celle des développements technologiques (nucléaire, renouvelables et dans une certaine mesure efficacité énergétique) révèle également une dichotomie entre des territoires dynamiques et des territoires évités. Le double mouvement de l'internationalisation des compagnies pétro-gazières, le plus souvent publiques, et du recours accru aux solutions technologiques étrangères modifie en profondeur la donne au sein du secteur énergétique indien. Alors que ce dernier se 
concentrait depuis des années sur l'autosuffisance, les déterminants de la demande ont obligé le pays à s'intégrer de plus en plus au marché mondialisé. Néanmoins le grand écart spatial toujours présent, en passe même de se creuser encore plus, risque d'aboutir à un éclatement territorial entre régions intégrées dans les flux mondiaux et espaces isolés, créant un risque potentiel sur l'unité nationale.

\section{BIBLIOGRAPHIE}

Ahn S., Graczyk D., 2012. Understanding Energy Challenges in India, Policies, Players and Issues. Paris, Agence Internationale de l'Énergie, 107 p.

Banque Asiatique de Développement, 2013. Key Indicators for Asia and the Pacific. Manille, BAD, 301 p.

Central Electric Authority, 2014. Load generation balance report 2014-2015. New Delhi, Government of India, $90 \mathrm{p}$.

Commission de la Planification, 2006. Integrated Energy Policy. New Delhi, Government of India, 148 p.

Dadwal S. R., 2009. India's Energy Security: Challenges and Opportunities. Eurasian Geography and Economics, vol. 50, n 6, p. 665-681.

Dhru K., Sivaram V., 2014. Can solar solve India's energy woes? World Economic Forum blog, en ligne, disponible à l'adresse: http://forumblog.org/2014/09/three-ways-india-can-get-best-solarpower/; consulté le 13/10/2014.

Energy Information Administration, 2014. India analysis brief, en ligne, disponible à l'adresse: www.eia.gov/countries/analysisbriefs/India/india.pdf; consulté le 13/10/2014.

Gutmann R., 2011. Le choix controversé du nucléaire en Inde. Etudes, 2011/9, Tome 415, p. $151-160$.

Gutmann R, 2011. Le nucléaire civil en Inde, Intérêts économiques et enjeux géopolitiques. Revue Tiers Monde, 2011/3, n 207, p. 25-41.

Lambert D., 2007. Géopolitique de l'Inde, védisme, laïcité et puissance nucléaire. Paris, Ellipses, 293 p.

Mallick H., 2009. Examining the Linkage between Energy Consumption and Economic Growth in India. The Journal of Developing Areas, Vol. 43, No. 1, p. 249-280.

Mazzucchi N., 2012. L'énergie, source de la nouvelle puissance brésilienne. Nouvelle Revue Géopolitique, $\mathrm{n}^{\circ}$ 3, avril-juin, p. 46-52.

Mistry D., 2006. Diplomacy, Domestic Politics, and the U.S.-India Nuclear Agreement. Asian Survey, Vol. 46, n5, September/October 2006, p. 675-698.

Tanvi M, 2006. The Brookings Foreign Policy Studies, Energy Security Series: India. Washington, Brookings, $98 \mathrm{p}$.

Vinod Kumar A., 2014. India's nuclear energy renaissance: stuck in the middle? Journal of Risk Research, vol. $17, \mathrm{n}^{\circ} 1$, p. 43-60. 


\section{NOTES}

1. Nécessité de disposer d'une capacité de production énergétique suffisante pour couvrir les besoins nationaux ou locaux.

2. Capacité à maintenir le niveau d'approvisionnement en énergie de manière constante et à un niveau satisfaisant.

3. L'Inde dépasse néanmoins la moyenne des émissions par habitants des pays d'Asie du Sud que la Banque Mondiale évalue à 1,4 tonne par habitant.

4. Le pays ne produit que 982000 barils par jour en 2013, moins du tiers de la demande nationale

5. Néanmoins dérivées de la technologie canadienne Candu (Pressurized Heavy Water Reactor ; PHWR) ne nécessitant pas ou peu d'enrichissement de l'uranium.

6. Deux réacteurs de $1000 \mathrm{MW}$ fournis par Rosatom, plus une option pour deux autres réacteurs de même puissance.

7. Il s'est notamment institué en réaction à l'alliance États-Unis - Pakistan.

8. L'Inde achète maintenant du combustible prêt à l'emploi majoritairement auprès de Rosatom, Kazatomprom et, de manière plus limitée, auprès d'Areva.

9. Suzlon est l'une des entreprises indiennes les plus mondialisées avec des centres de R\&D en Europe et des filiales en Amérique du Nord et dans le reste de l'Asie.

10. Entre autres par l'utilisation de centrales nucléaires et le développement des énergies renouvelables, installations bien moins émettrices par KWh. Selon le GIEC (annexe III du rapport $2014 \mathrm{du} 3 \mathrm{e}$ groupe de travail), les centrales à charbon $(760 \mathrm{~g})$ et à gaz $(370 \mathrm{~g})$ rejettent directement bien plus de $\mathrm{CO} 2$ par KWh que le nucléaire $(0)$ ou les renouvelables $(0 \mathrm{~g})$.

\section{RÉSUMÉS}

Le développement économique très rapide de l'Inde ces dernières années entraîne une modification de la problématique énergétique nationale. La volonté d'indépendance énergétique du pays se heurte de plus en plus à la hausse de la demande aussi bien en électricité qu'en carburant. Cette dernière oblige l'Inde à s'insérer de plus en plus dans les circuits du marché mondialisé en internationalisant ses compagnies pétrolières et en ayant de plus en plus recours aux technologies occidentales ou chinoises. Dans le même temps cette insertion crée une fragmentation au niveau national puisque certains États fédérés s'insèrent mieux que d'autres dans ce marché mondialisé, creusant un peu plus le fossé entre les régions les plus dynamiques et les espaces évités.

The energy situation of India is impacted by the intense economic development of the country for fifteen years. The goal of energy independence, center of India's energy policy, is continuously challenged with the growth of oil and electricity demand. As a consequence India is forced to enter the global competition by developing the national oil companies' international deployment as well as in using foreign - mostly Western and Chinese - energy technologies (nuclear, renewables, etc.). At the same time, globalization is creating a growing difference among Indian states as the most developed are thoroughly integrated and the poorest seem isolated from the global market and energy networks. 
INDEX

Mots-clés : Inde, énergie, pétrole, gaz, nucléaire, mondialisation

Keywords : India, energy, oil, gas, nuclear, globalization

Thèmes : Sur le Champ - Sur le Terrain

\section{AUTEUR}

\section{NICOLAS MAZZUCCHI}

Nicolas Mazzucchi, nicolas.mazzucchi@orange.fr est chercheur associé à l'IRIS et docteur en géographie. Il a publié récemment :

- Mazzucchi N., 2014. Les réseaux de pipelines en Asie centrale, effets d'une stratégie géoéconomique. In O. Kempf (dir.), Penser les réseaux, Paris, L'Harmattan ;

- Mazzucchi N., 2014. La manne énergétique russe, un vecteur géoéconomique d'influence au cœur des enjeux européens. Les grands dossiers de Diplomatie, n²1, avril 2014, p. 24-29. 